The Stereoselective Synthesis of a Potent Thrombin Inhibitor by a Novel P2-P3 Lactone

\title{
Ring Opening
}

Todd D. Nelson*, ${ }^{a}$ Carl R. LeBlond, ${ }^{a}$ Doug E. Frantz, ${ }^{a}$ Louis Matty, ${ }^{c}$ Jeffrey V. Mitten, ${ }^{a}$ Damian G. Weaver, ${ }^{a}$ Jeffrey C. Moore, ${ }^{b}$ Jaehon Kim, ${ }^{b}$ Russell Boyd, ${ }^{b}$ Pei-Yi Kim, ${ }^{b}$ Kodzo Gbewonyo, ${ }^{b}$ Mark Brower, ${ }^{b}$ Michael Sturr, ${ }^{b}$ Kathleen McLaughlin, ${ }^{b}$ Daniel R. McMasters, ${ }^{d}$ Michael H. Kress, ${ }^{a}$ James M. McNamara, ${ }^{c}$ Ulf H.Dolling ${ }^{c}$

${ }^{a}$ Department of Process Research, Merck Research Laboratories, Merck \& Co., 466 Devon Park Drive, Wayne, PA 19087

'Department of Bioprocess R\&D, Merck Research Laboratories, Merck \& Co., Rahway, NJ 07065

'Department of Process Research, Merck Research Laboratories, Merck \& Co., Rahway, NJ 07065

dDepartment of Molecular Systems, Merck Research Laboratories, Merck \& Co., West Point, PA 19486

\section{CONTENTS}

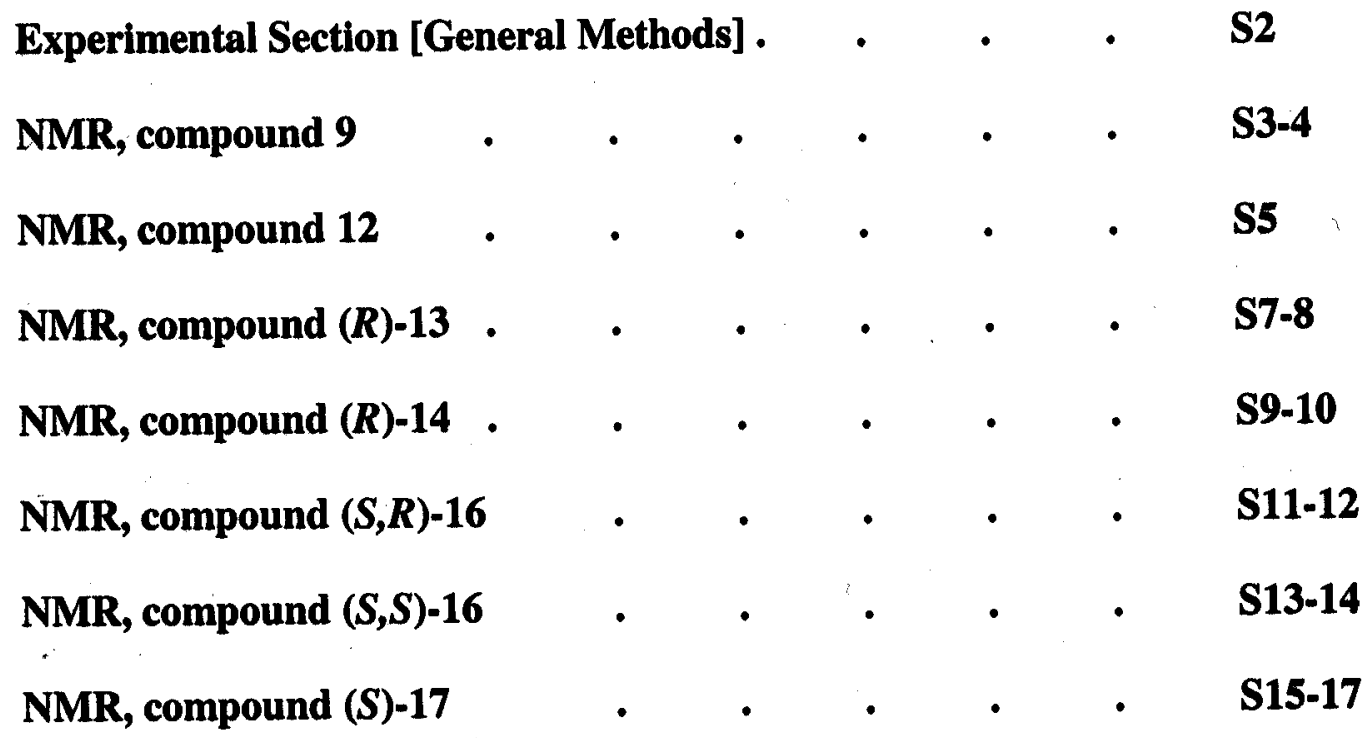




\section{Supporting Information}

\section{Experimental Section}

General Methods. The phosphate buffer stock solution was prepared as follows: $272.3 \mathrm{~g}$ of $\mathrm{K}_{2} \mathrm{HPO}_{4}$ was diluted with water to a final volume of $10 \mathrm{~L}$ while maintaining the $\mathrm{pH}$ at 7 with $50 \% \mathrm{v} / \mathrm{v} \mathrm{NaOH}$. This $200 \mathrm{mM}$ phosphate buffer solution was used to prepare the subsequent stock solutions: NADP solution (837 mg in $84 \mathrm{~mL}$ of 200 phosphate buffer solution); glucose solution (800 g in $2 \mathrm{~L}$ of phosphate buffer solution); GDH (3.51 $\mathrm{g}$ in $350 \mathrm{~mL}$ of 200 phosphate buffer solution); KRED-1001 (750 $\mathrm{mg}$ in $15 \mathrm{~mL}$ of buffer solution). LCAP refers to HPLC area percent and LCWP refers to HPLC weight percent. Racemic 13 was prepared by $\mathrm{NaBH}_{4}$ reduction of ketoester 12 for chiral GC development: determined by chiral GC: CyclosilB, $30 \mathrm{~m} \times 0.25 \mathrm{~mm} \mathrm{ID} \times 0.25 \mathrm{~mm}$ film thickness, isothermal $80^{\circ} \mathrm{C}, 1 \mu \mathrm{L} / \mathrm{min}$ He. Racemic hydroxy acid 14 was prepared by the saponification of racemic hydroxy ester 13 for chiral HPLC development: ChiralPak AD-H, $150 \mathrm{~mm} \times 4.6 \mathrm{~mm}$; 85/15/0.1 hexane/EtOH/TFA, flow rate 1.5 $\mu \mathrm{L} / \mathrm{min}$. Methyl-2-bromo-5-chlorobenzoate was purchased from Esprit Chemical Co., Sarasota, FL 34243. KRED1001 was purchased from BioCatalytics, Pasadena, CA 91106. 3,3-Dimethyl-2-oxobutanoic acid was purchased from either Polycarbon Inc., Leominster, MA 01453 or Alfa Aesar, Ward Hill, MA 01835. 
$d p x-300 \quad c-13$

g

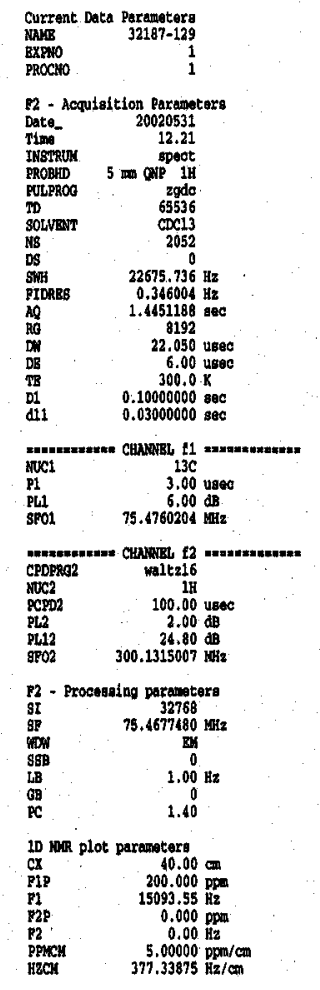

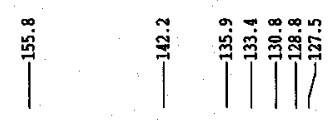<smiles>NCc1cc(Cl)ccc1CNC(=O)O[Na]</smiles>

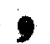

فำ

${ }^{13} \mathrm{C} \mathrm{NMR} \mathrm{(75.5} \mathrm{MHz)} \mathrm{in} \mathrm{CDCl}_{3}$

$\|\left.^{\infty \circ}\right|^{n}$ 
是

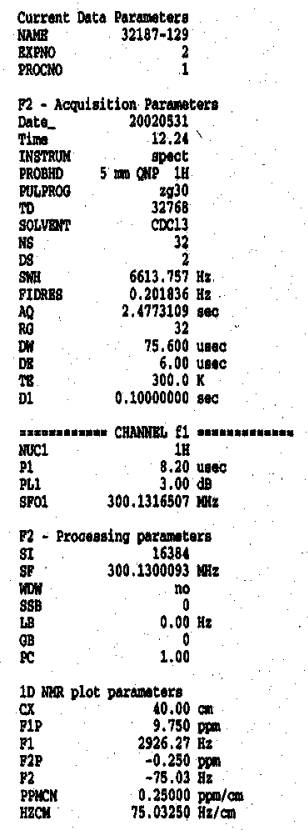

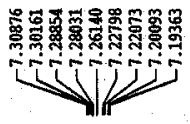

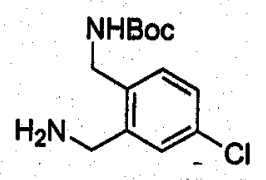

9
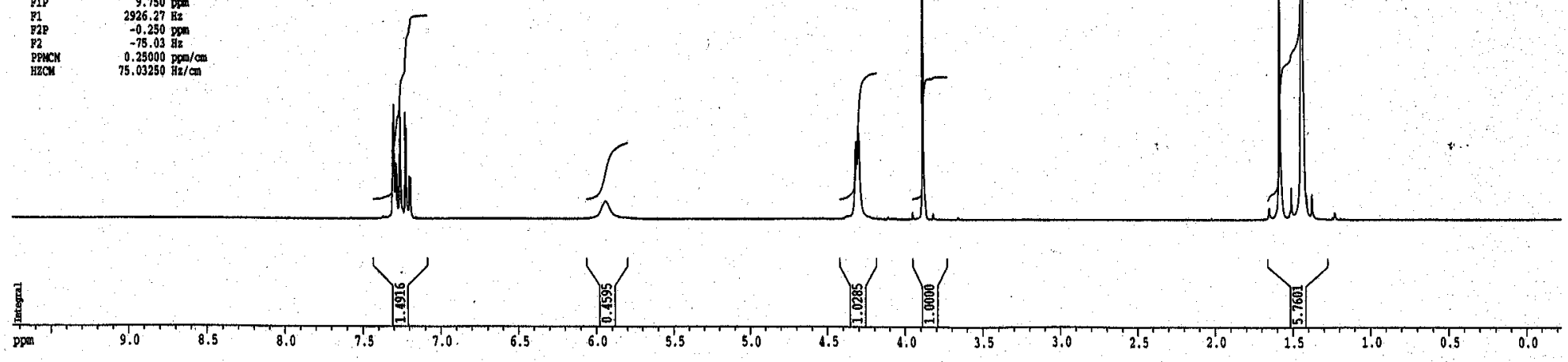

嵒零

${ }^{1} \mathrm{H} \mathrm{NMR} \mathrm{(300} \mathrm{MHz)} \mathrm{in} \mathrm{CDCl}_{3}$

哰蛋

$d p x-300 \cdot h-1$
墨

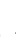

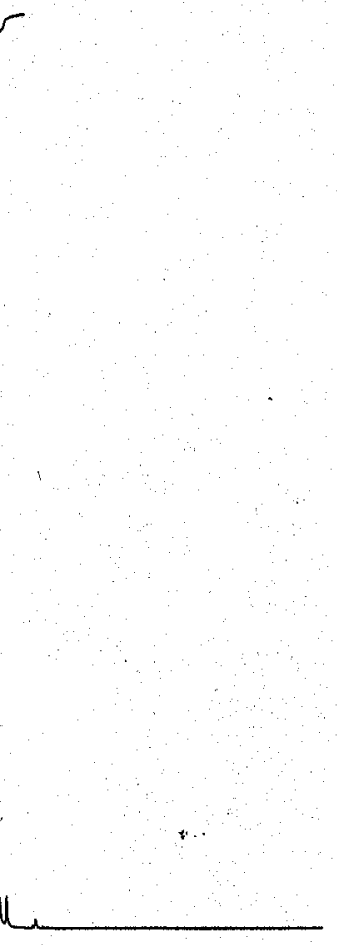

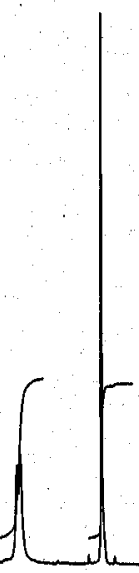

54 


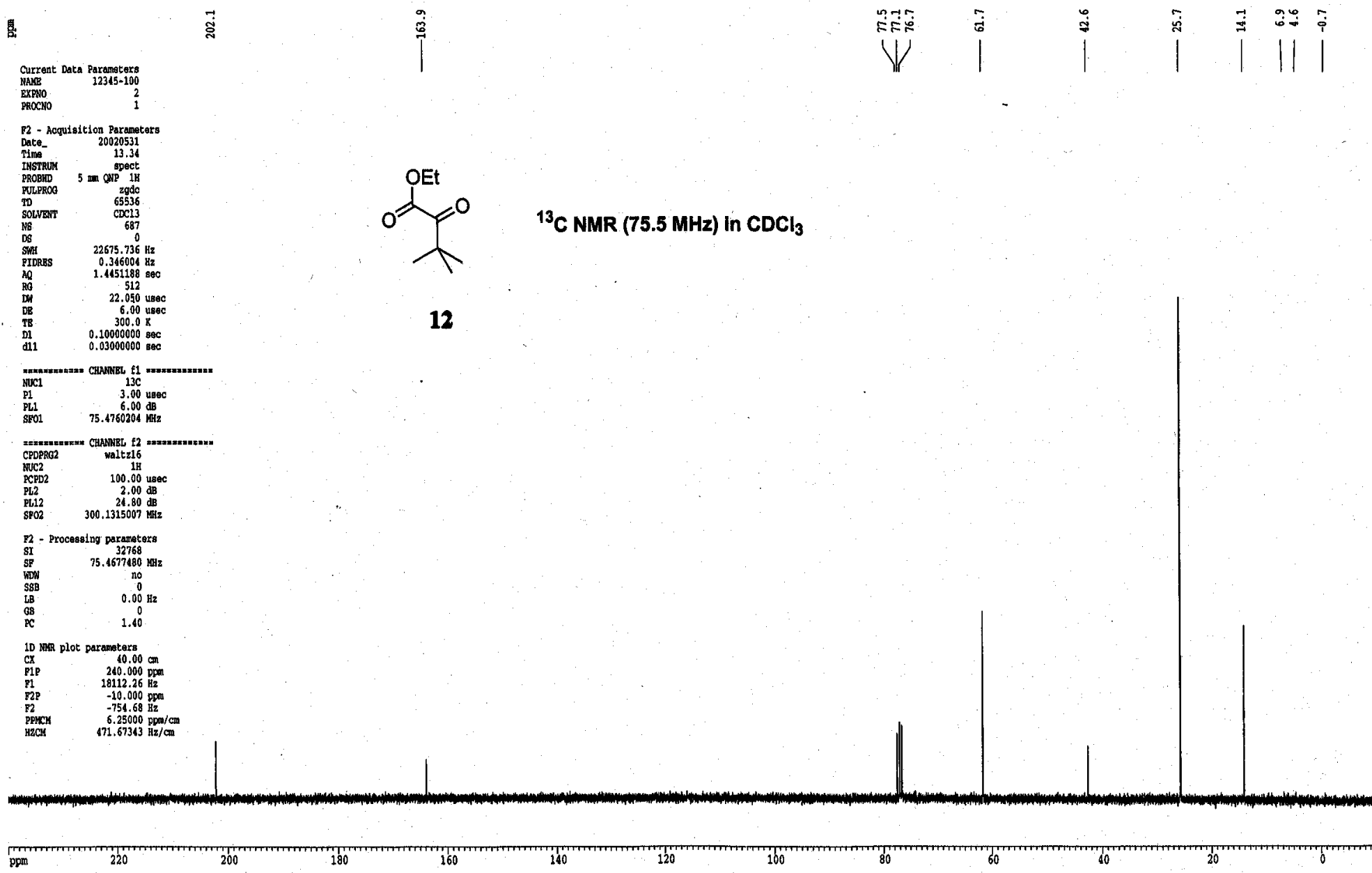


s
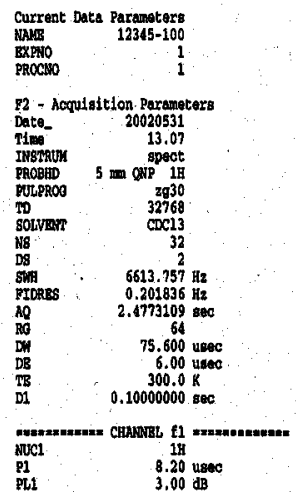

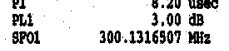

D2 - Procosasing paranotera

$\begin{array}{rr}\mathrm{gI} & 16384 \\ \mathrm{gP} & 300.1300093 \mathrm{.Hz}\end{array}$

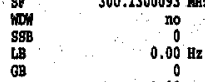

1D NIR plot paranaters

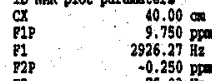

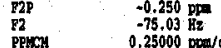

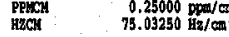<smiles>CCOC(=O)C(=O)C(C)(C)C</smiles>

13

${ }^{1} \mathrm{H}$ NMR (300 MHz) In $\mathrm{CDCl}_{3}$

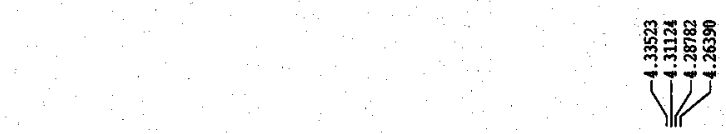

్ㅗㅀㅁㅕำ

$d p x-300 h-1$
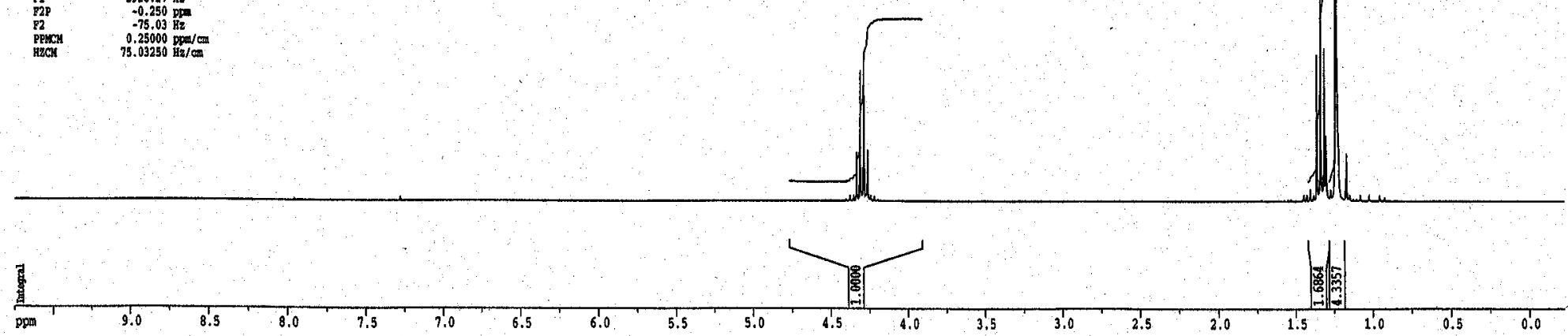
동

Current Data Parameters
MARB
BXPNO
B2345-101

EXPNO
PROCFO

F2 - Aequiaition Paranate

Date- 20020531

.

TD

NS

$\begin{array}{ll}\text { PIDRES } & 0.346004 \mathrm{~Hz} \\ 10 & 1.3451198\end{array}$

512

22.050 usec
6.00 ugec

10000000

0.100000000
0.030000000
8006

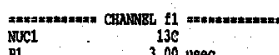

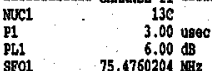

CEDPRG2

$\begin{array}{lc}\text { CFDPRG2 } & \text { waltz16 } \\ \text { MIOC2 } & 1 \mathrm{~B} \\ \text { PCPD2 } & 100.00 \text { usec } \\ \text { PC2 } & 2.00 \mathrm{~dB}\end{array}$

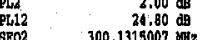

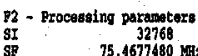

WF
SSB

$\begin{array}{ll}48 & 1.00 \mathrm{~Hz} \\ G \mathrm{~B} & 1.40 \\ \mathrm{XC} & 1.40\end{array}$

10 MRR plot paramatere

FIP $\quad 210.000 \mathrm{~cm}$

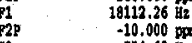

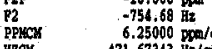

$6.25000 \mathrm{ppot} / \mathrm{cm}$
$471.67343 \mathrm{~Hz} / \mathrm{cm}$ $\stackrel{n}{3}$<smiles>CCOC(=O)C(O)(C(C)(C)C)C(C)(C)C</smiles>

${ }^{13} \mathrm{C} \mathrm{NMR} \mathrm{(75.5} \mathrm{MHz)} \mathrm{in} \mathrm{CDCl}_{3}$
ํㅜㅇำ

IV

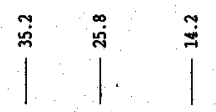

\section{(a)-13}

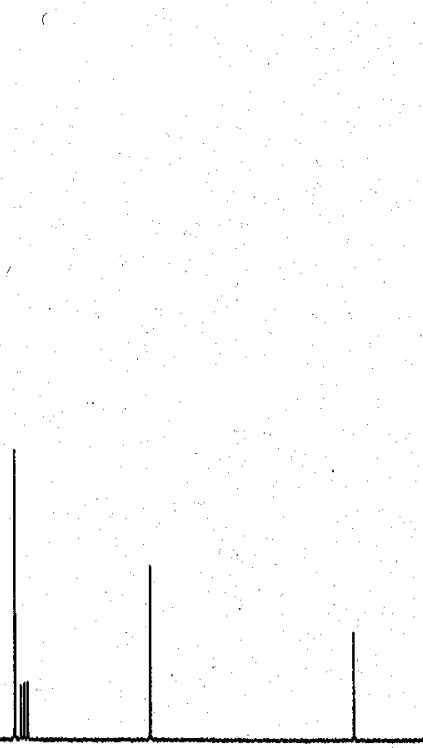

ppm 220

200

160

140

100

80

60

40

${ }_{20}$

T) 
2 - Acquigitton paranater

Date_ 20020531

MISTROM 5 un GNP 1I

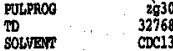

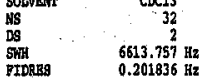

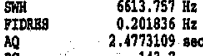

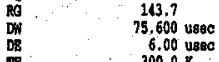

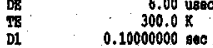

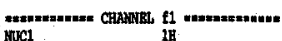

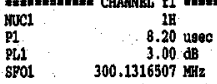<smiles>CCOC(=O)C(O)(C(C)(C)C)C(C)(C)C</smiles>

${ }^{1} \mathrm{H}$ NMR (300 MHz) In $\mathrm{CDCl}_{3}$

F2 - procensing paranetors

$\underset{\mathrm{SI}}{\mathrm{PL}}-\mathrm{Procosasing}$ paranaters

$\begin{array}{cc}\text { IDW } & 300.1300093 \\ \text { SAB } & 0 \\ \mathrm{LB} & 0.00 \mathrm{~Hz}\end{array}$

$0 \mathrm{~B}$
$\mathrm{PC}$
1.00

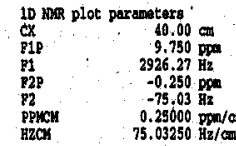

\section{(a)-13}

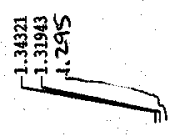

$\underset{\infty}{\infty}$
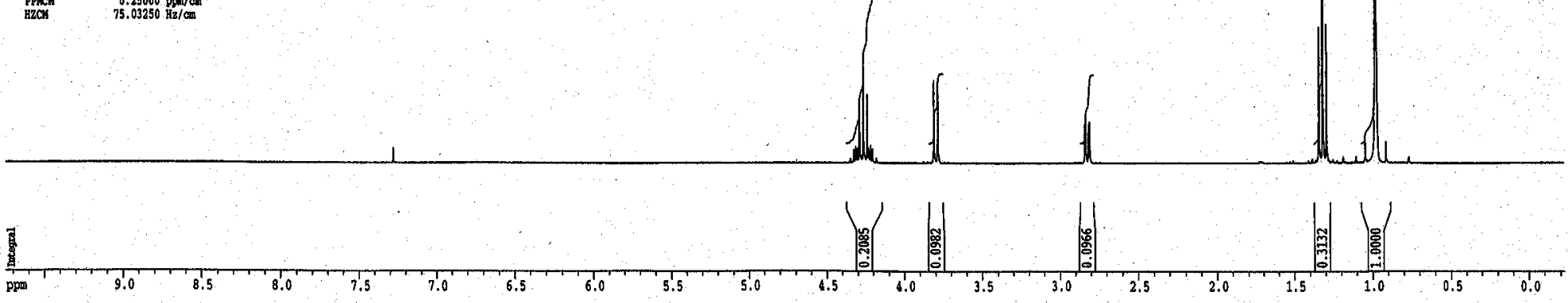
82 - Acqulaition Parapotors

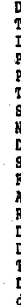<smiles>CC(C)(C)[C@@H](O)C(=O)O</smiles>

${ }^{13} \mathrm{C} \mathrm{NMR} \mathrm{(75.5} \mathrm{MHz)} \mathrm{in} \mathrm{CDCl}_{3}$

\section{(1)- 14 \\ NTC2 Maltzit6}

(3)

100.00
0.000

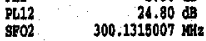

$\frac{\mathrm{g} 2}{\mathrm{gI}}-$ Procesesing paranatars

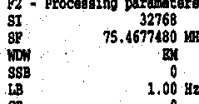

$\mathrm{OB}$
$\mathrm{BC}$

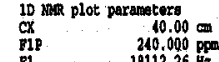

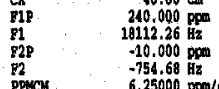

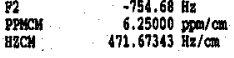

\section{}

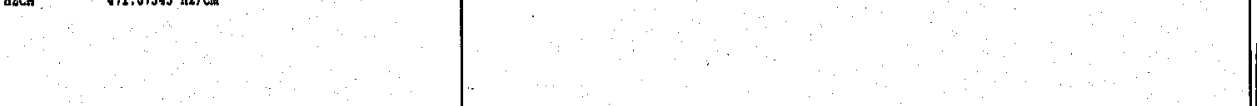

${ }_{\mathrm{ppm}}$

180

160

140

120

100

80

20 
5

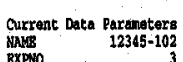

$\begin{array}{ll}\text { RXENO } & 3 \\ \text { PROCNIO } & \therefore\end{array}$

Pn:- Aequisition Parangtero
Date- 20020531

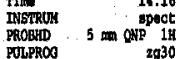

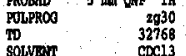

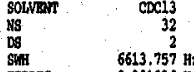

$\begin{array}{ll}\text { SHA } & 6613.757 \mathrm{~Hz} \\ \text { FDPRES } & 0.201836 \mathrm{~Hz}\end{array}$

$\mathrm{AQ}$
$\mathrm{ng}$$\quad \therefore .7773109 \mathrm{800}$

75.600 uave
6.00 usec
$300.0 \mathrm{R}$

0.10000000 800

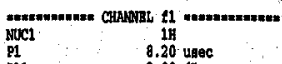

Q61 $\quad 3.2000$

SE01 $\quad 300.1316507$ tat

82 - Processing paramaters

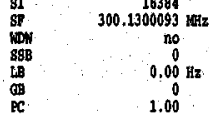

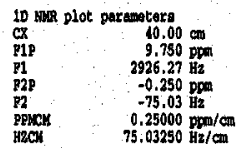

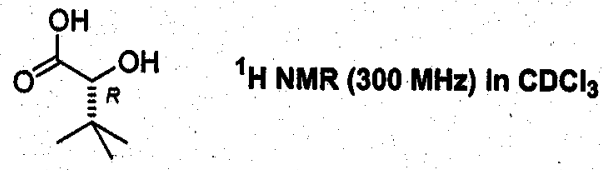

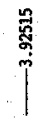

\section{(a)-14}

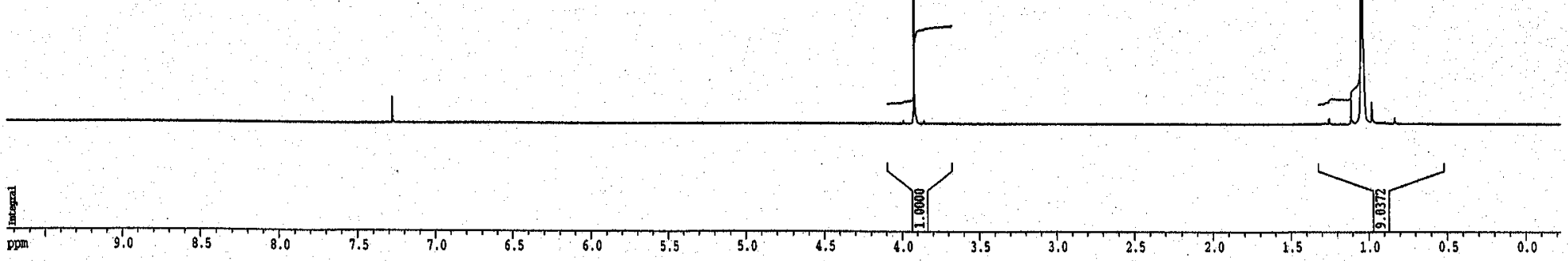


量

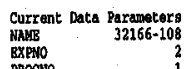

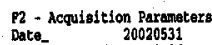

Date-
rime
rime

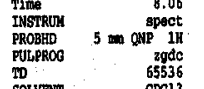

$\begin{array}{ll}\text { TD } & 65536 \\ \text { Sovvarn } & \text { CDc13 } \\ \text { Ns } & 1801 \\ \text { Ds } & 0\end{array}$

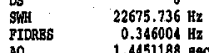

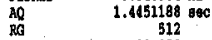

22.050 uasec

6.00 480

$0.1000000 \mathrm{gaC}$
$0.03000000 \mathrm{sec}$

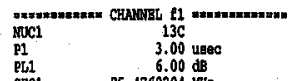

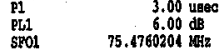

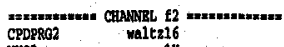

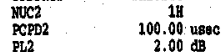

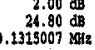

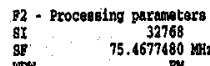

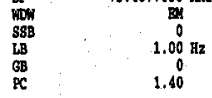

10 MMR plot peransecers

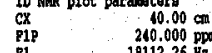

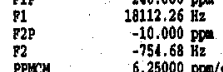

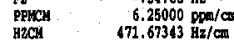

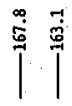

(2)

|

${ }^{13} \mathrm{C}$ NMR (75.5 MHz) in $\mathrm{CDCl}_{3}$

\section{(S,R)-16}

ppm 

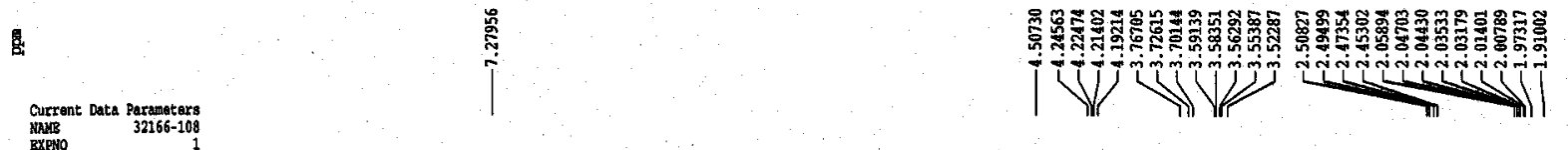

$\mathrm{dpx}-300 \mathrm{~h}-1$

P2 - Acquidit ten parameters

Date- 20020351.

IIISTRU

PROOI:D
PULPROO

SOLVBM
NS
DS
S

$\quad 23$

$6513.757 \mathrm{~Hz}$
$0.201936 \mathrm{~Hz}$

2. 6773109

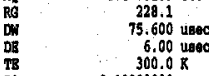

0.1000000000

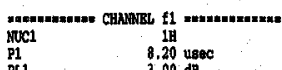

$8.20 \mathrm{use}$
$\mathrm{PC1}$$\quad 3.00 \mathrm{~dB}$

Spo1 $\quad 300.1316507 \mathrm{kHz}$

P2 - Procasaing paramaters

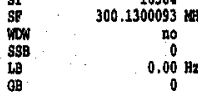

0.00
0.0 $\quad 1.00$

ID MIMR plot paraminter: $40.00 \mathrm{~cm}$

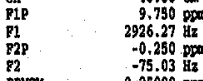

PPHCH : $\quad 0.25000 \mathrm{ppan} / \mathrm{cos}$

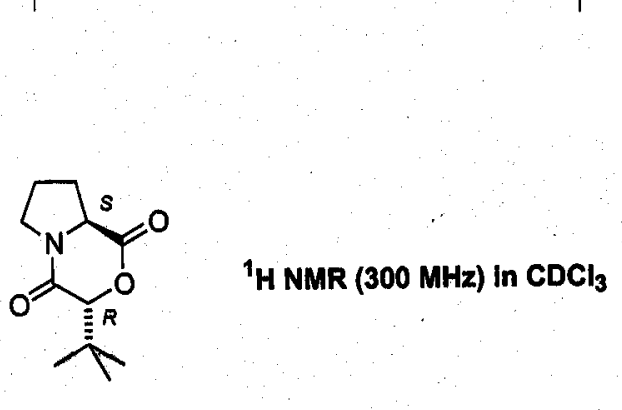

$(9, n)-16$

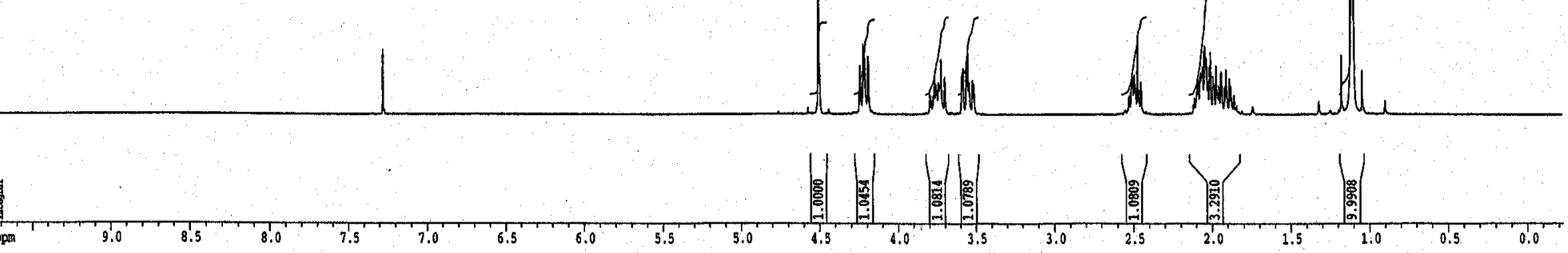


E

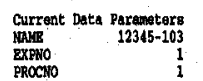

P2 - Acquidetitlon Paranatere

Data-
Tinas

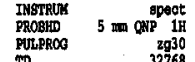

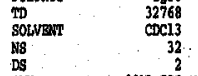

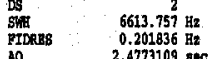

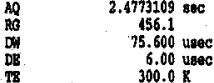

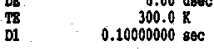

HOMCI

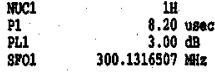

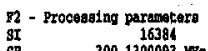

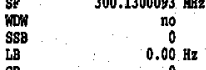

${ }_{\mathrm{PC}}^{\mathrm{ag}} \quad 1.00$

10 NRR plot paranatars 40.00

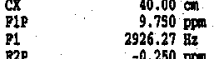

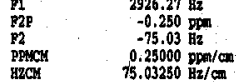

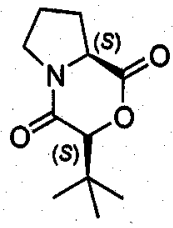

${ }^{1} \mathrm{H}$ NMR (300 MHz) In $\mathrm{CDCl}_{3}$

\section{(6) 16}

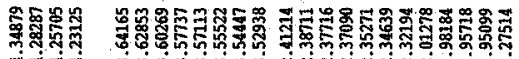

Jij

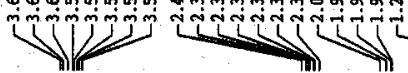

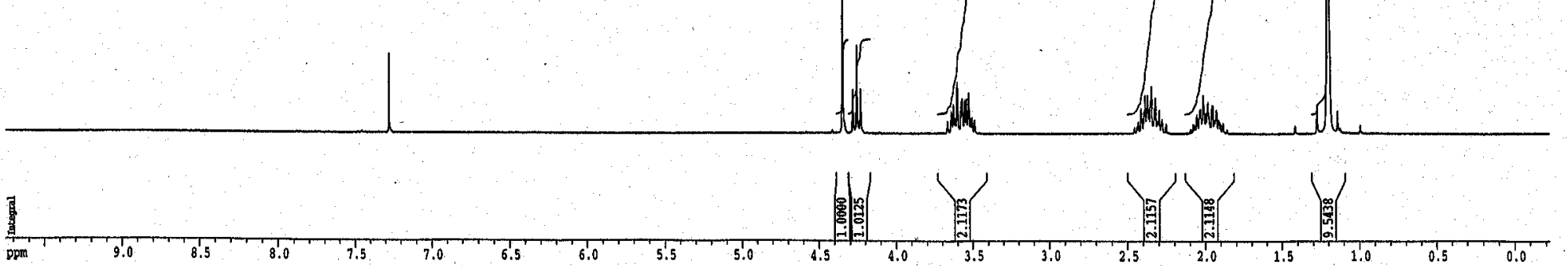



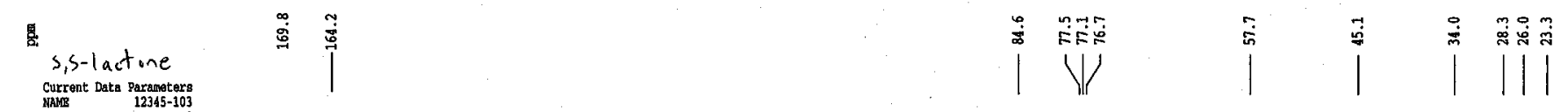

Current Data Paramaters
NAMT
RXPYO
PROCNO

F2 - Accuigitt on Paranetora
Date_- 20020531

$\begin{array}{lr}\text { Date- } & 20020531 \\ \text { Time } & 14.57\end{array}$

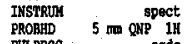

$\begin{array}{lr}\text { PULPROG } & \text { ggde } \\ \text { TD } & 65536 \\ \text { SOLVAII } & \text { COC13 } \\ \text { NS } & 644 \\ \text { DS } & \text { SA }\end{array}$

$\begin{array}{ll}\text { NS } & 640 \\ \text { DS } & 02675.736 \mathrm{~Hz} \\ \text { SMH } & 0.3600 \text {. }\end{array}$

$\begin{array}{ll}\text { PIDRES } & 0.346004 \mathrm{~Hz} \\ \mathrm{AO} & 1.445118 \mathrm{seC}\end{array}$

$\begin{array}{rr}\mathrm{AO} & 1.4451188 \mathrm{sec} \\ \mathrm{RQ} & 512 \\ \mathrm{RO} & 22.050 \text { usec } \\ \mathrm{DE} & 6.00 \text { usec } \\ \mathrm{DE} & 300.0 \mathrm{~g}\end{array}$

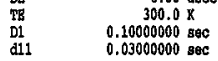

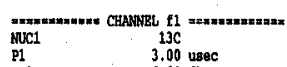

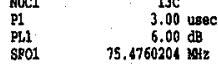

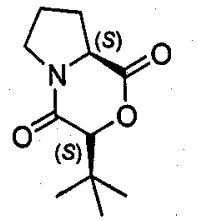

${ }^{13} \mathrm{C}$ NMR $\left(75.5 \mathrm{MHz}\right.$ ) in $\mathrm{CDCl}_{3}$

$(S, S)-16$

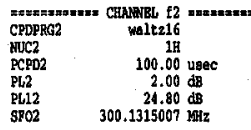

P2 - Processing parameters

$\begin{array}{cc}\mathrm{SI} & 752768 \\ \mathrm{SP} & 75.4677480 \\ \mathrm{WDW} & \mathrm{BP} \\ \mathrm{SSB} & 0 \\ \mathrm{IB} & 1.00 \mathrm{~Hz} \\ \mathrm{~GB} & 0 \\ \mathrm{OC} & 1.0\end{array}$

1D MRR plot paranaters

$\begin{array}{lr}\mathrm{CX} & \begin{array}{l}40.00 \mathrm{~cm} \\ \mathrm{PIP}\end{array} \\ \mathrm{F1} & 15093.55 \mathrm{pgm}\end{array}$

$\begin{array}{ll}\mathbf{P 2 P} & 0.000 \mathrm{ppm} \\ \mathbf{P 2} & 0.00\end{array}$

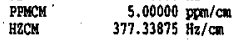
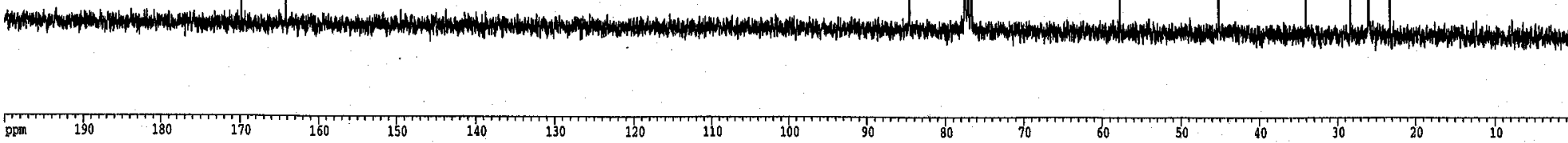


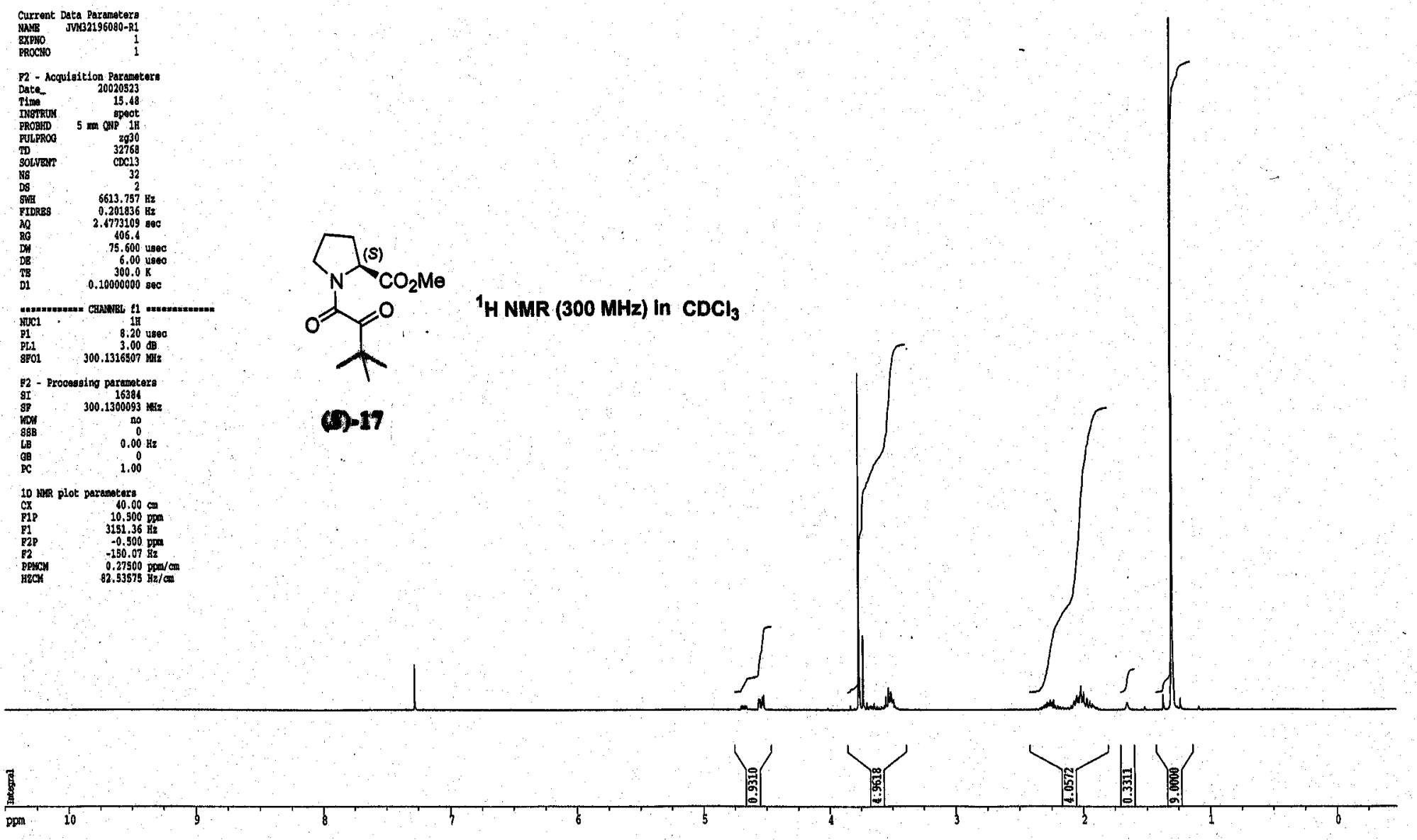




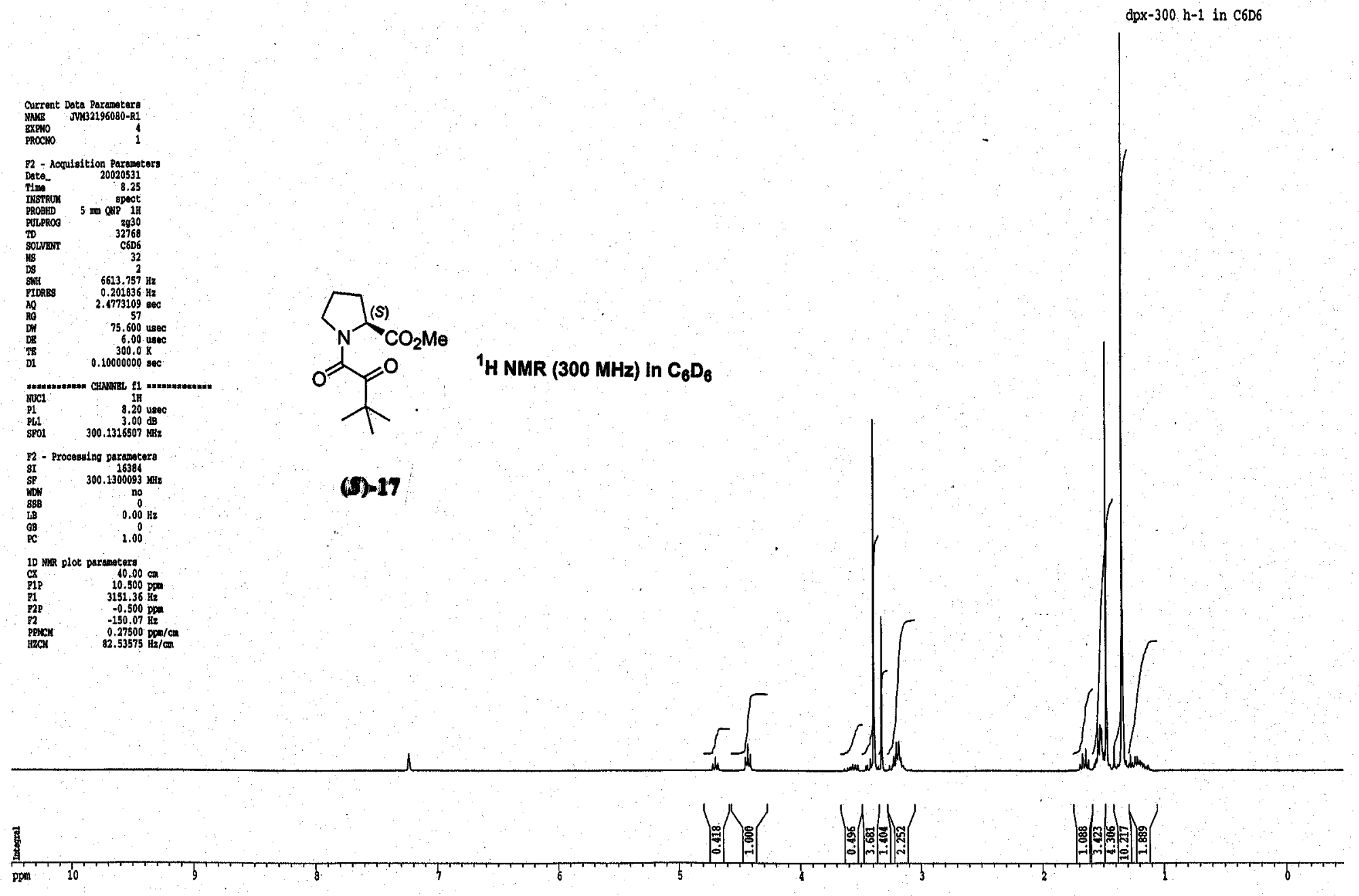

dpx-300 $h-1$ in C6D6 

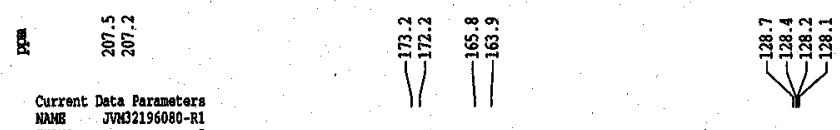

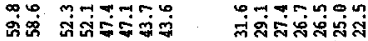

IIVV

livil

NAMER
EXRONO
RROCNO

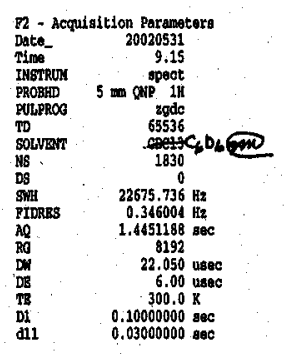

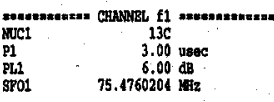

$\bigcap_{\mathrm{N}}(\mathrm{s})$<smiles>CC(=O)C(=O)C(C)(C)C</smiles>

${ }^{13} \mathrm{C}$ NMR (75.5 MHz) in $\mathrm{C}_{6} \mathrm{D}_{6}$

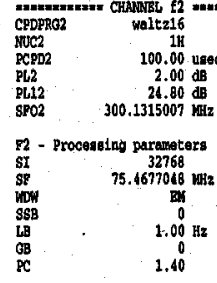

1D MER plot pararators

$\begin{array}{cc}\text { P1P } & 220.000 \\ 71 & 16602.89 \\ 72 P & -10.000 \\ 72 & 756.68\end{array}$

$\begin{array}{ll}\text { PPyct } & 5.75000 \\ \text { HzCH } & 433.93930 \mathrm{~Hz} / \mathrm{cm}\end{array}$

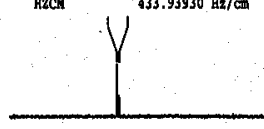

(S)-17

ppen

200

180

160

140

120

100 